\title{
Overoxidation of chloroplast 2-Cys peroxiredoxins: balancing toxic and signaling activities of hydrogen peroxide
}

\author{
Leonor Puerto-Galán ${ }^{1}$, Juan M. Pérez-Ruiz ${ }^{1}$, Julia Ferrández ${ }^{1}$, Beatriz Cano ${ }^{1}$, Belén Naranjo ${ }^{1}$, \\ Victoria A. Nájera' ${ }^{1}$, Maricruz González ${ }^{1}$, Anna M. Lindaht ${ }^{2}$ and Francisco J. Cejudo ${ }^{1 *}$
}

${ }^{1}$ Instituto de Bioquímica Vegetal y Fotosíntesis, Universidad de Sevilla, Sevilla, Spain

${ }^{2}$ Consejo Superior de Investigaciones Científicas, Sevilla, Spain

\section{Edited by:}

Jose A. Traverso, Consejo Superior

de Investigaciones Científicas, Spain

\section{Reviewed by:}

Sang Yeol Lee, Gyeongsang National University, South Korea

Juan José Lázaro, Consejo Superior de Investigaciones Científicas, Spain

\section{*Correspondence:}

Francisco J. Cejudo, Instituto de Bioquímica Vegetal y Fotosíntesis, Universidad de Sevilla, Avenida Américo Vespucio 49, 41092 Sevilla, Spain

e-mail: fjcejudo@us.es
Photosynthesis, the primary source of biomass and oxygen into the biosphere, involves the transport of electrons in the presence of oxygen and, therefore, chloroplasts constitute an important source of reactive oxygen species, including hydrogen peroxide. If accumulated at high level, hydrogen peroxide may exert a toxic effect; however, it is as well an important second messenger. In order to balance the toxic and signaling activities of hydrogen peroxide its level has to be tightly controlled. To this end, chloroplasts are equipped with different antioxidant systems such as 2-Cys peroxiredoxins (2-Cys Prxs), thiol-based peroxidases able to reduce hydrogen and organic peroxides. At high peroxide concentrations the peroxidase function of 2-Cys Prxs may become inactivated through a process of overoxidation. This inactivation has been proposed to explain the signaling function of hydrogen peroxide in eukaryotes, whereas in prokaryotes, the 2-Cys Prxs of which were considered to be insensitive to overoxidation, the signaling activity of hydrogen peroxide is less relevant. Here we discuss the current knowledge about the mechanisms controlling 2-Cys Prx overoxidation in chloroplasts, organelles with an important signaling function in plants. Given the prokaryotic origin of chloroplasts, we discuss the occurrence of 2-Cys Prx overoxidation in cyanobacteria with the aim of identifying similarities between chloroplasts and their ancestors regarding their response to hydrogen peroxide.

Keywords: chloroplast, hydrogen peroxide, peroxiredoxin, redox regulation, thioredoxin, oxidative stress

\section{INTRODUCTION}

Oxygenic photosynthesis is an essential process for life on Earth because it allows the use of light and water to produce biomass and oxygen. However, it is also a process potentially harmful due to the transport of electrons in the presence of oxygen, which inevitably produces reactive oxygen species (ROS). Several environmental challenges such as drought, low or high temperature, high light intensity, or salinity, alter chloroplast ROS homeostasis producing oxidative stress (Miller et al., 2010). To adequately respond to these stressful conditions chloroplasts are equipped with different antioxidant systems both enzymatic and non-enzymatic. It should be taken into account that besides their harmful effect, ROS have also signaling function (Laloi et al., 2007). This is the case of hydrogen peroxide, which is produced at high rate in chloroplasts of photosynthetic cells and has an important signaling activity (Mubarakshina et al., 2010), as confirmed by genome-wide expression analyses in tobacco and Arabidopsis (Vandenabeele et al., 2003; Vanderauwera et al., 2005).

Peroxiredoxins (Prxs), thiol-based peroxidases able to reduce hydrogen peroxide, peroxynitrite and organic peroxides, are among the most abundant chloroplast enzymatic antioxidant systems. Prxs are universally present in any type of organisms from bacteria to animals and plants (Dietz, 2003, 2011; Wood et al., 2003a; Rhee etal., 2005; Hall etal., 2009). These enzymes are classified into different classes including typical 2-Cys Prxs, which are homodimeric, atypical 2-Cys Prxs, which are monomeric, and 1-Cys Prxs. Both typical and atypical 2-Cys Prxs share a similar reaction mechanism involving two conserved Cys residues, termed peroxidatic and resolving, respectively (Wood et al., 2003a; Hall et al., 2009). During the catalytic cycle the peroxidatic Cys becomes transiently oxidized as sulfenic acid (-SOH) and then condenses with the resolving Cys to form a disulfide bridge. In the case of the typical 2-Cys Prxs, which are dimeric, the enzyme is fully oxidized when both pairs of catalytic Cys residues (peroxidatic and resolving) form disulfides, which have to be reduced to initiate a new catalytic cycle. This reduction is performed by a thiol-oxidoreductase, which usually is thioredoxin (Trx), though glutaredoxin and cyclophilins are also able to participate in this step. At high peroxide concentrations, the sulfenic acid intermediate of the peroxidatic Cys may become overoxidized to sulfinic $\left(-\mathrm{SO}_{2} \mathrm{H}\right)$ or even sulfonic $\left(-\mathrm{SO}_{3} \mathrm{H}\right)$ acids, which causes the inactivation of the enzyme. Based on the different sensitivities of 2-Cys Prxs to overoxidation, which is higher in enzymes from eukaryotes than from prokaryotes, Wood et al. (2003b) proposed the floodgate hypothesis. According to this hypothesis, oxidizing conditions promote the inactivation by overoxidation of sensitive 2-Cys Prxs in eukaryotic organisms, provoking a transient further increase of hydrogen peroxide, which may then be used as second messenger 
(Wood et al., 2003b). In contrast, in prokaryotic organisms, the 2-Cys Prxs of which were considered to be insensitive, hydrogen peroxide is efficiently reduced and does not accumulate, thus having a less important function in signaling. Different reports confirm the relevant role of the hydrogen peroxide-dependent inactivation of 2-Cys Prxs in signaling processes in eukaryotic organisms (Karplus and Poole, 2012; Rhee et al., 2012). A notion reinforced by the recent finding that 2-Cys Prx overoxidation is a conserved marker of circadian rhythmicity (Edgar et al., 2012).

In plants, Prxs are encoded by a gene family, which in Arabidopsis is composed of ten members (Dietz, 2003). The first plant Prx identified was a 1-Cys Prx highly expressed in barley grains (Stacy et al., 1996). Later it was shown that this 1-Cys Prx accumulates in the nucleus of cereal seed tissues that undergo intense oxidative stress (Stacy et al., 1999; Pulido et al., 2009), suggesting a function in the antioxidant protection of nuclear structures. Chloroplasts are the organelles with the highest content of Prxs. The Arabidopsis chloroplast contains two almost identical typical 2-Cys Prxs, termed A and B, and atypical monomeric Prxs Q and IIE (Dietz, 2003, 2011). Although 2-Cys Prxs are among the most abundant plastidial proteins, a double mutant of Arabidopsis, which is a severe knock down for 2-Cys Prxs, shows a surprisingly mild phenotype (Pulido et al., 2010) suggesting that other antioxidant systems, such as the ascorbate-glutathione cycle in combination with superoxide dismutase, are able to compensate for 2-Cys Prx deficiency. Moreover, it was shown that chloroplast 2-Cys Prxs are sensitive to overoxidation, hence behaving as eukaryotic-type enzymes despite the endosymbiont origin of this organelle (Kirchsteiger et al., 2009). A more in-depth analysis of 2-Cys Prx in cyanobacteria showed that the enzyme from Anabaena is more sensitive to overoxidation than the Synechocystis enzyme (Pascual et al., 2010).

In this review we will discuss our present knowledge of the mechanisms controlling 2-Cys Prxs reduction and overoxidation in chloroplasts with emphasis in the effect of the redox status of 2-Cys Prxs on the activity of these enzymes. Moreover, we will discuss how the redox status of the chloroplast influences the signaling function of this organelle, which is essential to harmonize the growth and development of the different plant organs.

\section{THE PATHWAYS OF 2-Cys Prxs REDUCTION IN CHLOROPLASTS}

The Arabidopsis chloroplast is equipped with two almost identical typical 2-Cys Prxs, A and B, and atypical Prxs Q and IIE (Dietz et al., 2006). Although all these enzymes are relatively abundant, 2-Cys Prxs are among the most abundant proteins of the chloroplast (Dietz, 2011). Concerning their suborganellar localization, the presence of Prx IIE in the chloroplast stroma has been established, though its interaction with internal membranes was not analyzed (Bréhélin et al., 2003). Prx Q, which was described initially as associated to thylakoids (Lamkemeyer et al., 2006), was later localized in the thylakoid lumen (Petersson et al., 2006). Therefore, the exact localization of Prx Q still awaits confirmation (Dietz, 2011). 2-Cys Prxs A and B are localized in the chloroplast stroma in dimeric form, but become associated to the thylakoid membrane in their oligomeric form (Konig et al., 2002).
The function of the chloroplast-localized Prxs was first addressed by the generation of transgenic plants with antisense suppression of 2-Cys Prx expression (Baier et al., 2000), and then by the analysis of Arabidopsis mutants (Pulido et al., 2010). Single mutants with reduced levels of 2-Cys Prx A or lacking 2-Cys Prx B showed no phenotypic differences as compared with wild type plants, suggesting redundant functions of these enzymes (Pulido et al., 2010). Surprisingly, the double mutant $\Delta 2 c p$, which is knock out for 2-Cys Prx B and a severe knock down for 2-Cys Prx A, shows almost wild type phenotype (Pulido et al., 2010). However, it was not possible to obtain a double knock out mutant, which suggests that plants cannot survive without at least a small amount of chloroplast 2-Cys Prxs. Most probably this is not exclusively due to their peroxidase activity, which can be compensated for by the other antioxidant systems of the chloroplast, such as the ascorbate-glutathione cycle in combination with superoxide dismutase. 2-Cys Prxs are complex enzymes showing different activities associated with different quaternary structures. As initially shown for the enzyme from yeast (Jang et al., 2004), the low-molecular-weight (LMW) form of 2-Cys Prxs shows predominantly peroxidase activity, whereas higher molecular weight (HMW) forms lack peroxidase activity while gaining chaperone activity. Interestingly, the switch from LMW to HMW is triggered under conditions of oxidative stress.

Chloroplast 2-Cys Prxs have a reaction mechanism similar to the enzyme from other eukaryotic organisms. The LMW form of the enzyme is arranged as a head-to-tail homodimer, which in its reduced form displays peroxidase activity (Figure 1). Therefore, 2-Cys Prxs can be considered as symmetric enzymes having two identical active sites. The catalytic cycle is initiated by the attack of the peroxidatic Cys to the peroxide rendering the corresponding alcohol, or water in the case of hydrogen peroxide, and the Cys residue oxidized to sulfenic acid (Figure 1). In a second step, the sulfenic acid intermediate is condensed with the resolving Cys producing a molecule of water and both Cys linked by a disulfide bridge (Figure 1). For a new catalytic cycle this disulfide has to be reduced. In chloroplasts Broin et al. (2002) proposed that a previously identified protein, termed CDSP32, which is formed by two Trx folds, with only one of them harboring a Trx active site, acted as reductant of 2-Cys Prxs. The in vitro analysis of several plastidial Trxs led Collin et al. (2003) to propose $\operatorname{Tr} x$ as the most efficient reductant of these enzymes. Finally, the chloroplast localized NADPH-dependent thioredoxin reductase C (NTRC), a peculiar NTR with a joint Trx domain at the C-terminus (Serrato et al., 2002, 2004) was shown to combine both NTR and Trx activity to efficiently reduce plastidial 2-Cys Prx (Moon et al., 2006; Pérez-Ruiz et al., 2006; Alkhalfioui et al., 2007). The notion that NTRC is the most efficient reductant of chloroplast 2-Cys Prxs was subsequently confirmed in further studies by in vivo analysis, based on fluorescence resonance energy transfer (FRET) assays, which showed interaction of 2-Cys Prx with NTRC but not with $\operatorname{Trx} x$ (Muthuramalingam et al., 2009). In addition, the redox status of 2-Cys Prxs was similar in wild type and $\operatorname{Trx} x$ knock out mutant plants, whereas the ntrc mutant showed a severely impaired redox status (Pulido et al., 2010). In summary, as depicted in Figure 1, chloroplast 2-Cys Prxs have a mode of action similar to the 


\section{Light

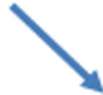 \\ Fd $\rightleftharpoons$ NADPH

 \\ $\operatorname{Trx} x$ \\ $\operatorname{CDSP} 32$}
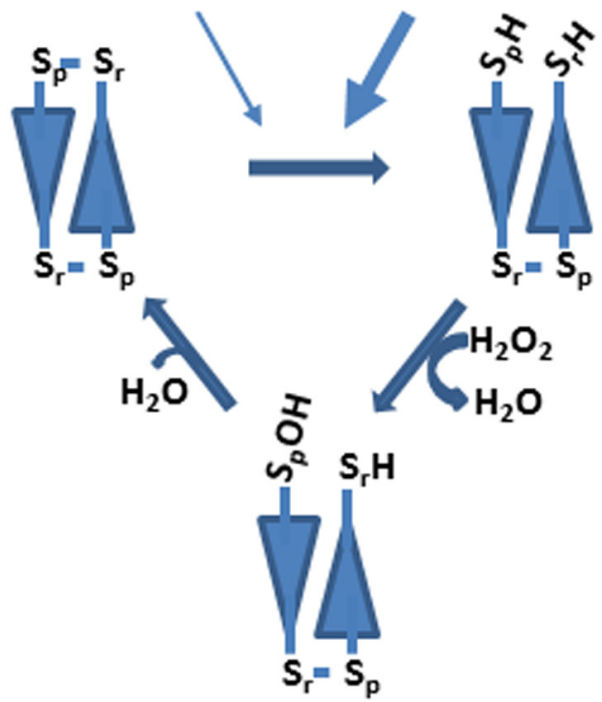

FIGURE 1 | Reaction mechanism and pathways for 2-Cys Prx reduction in chloroplasts. Typical 2-Cys Prxs are homodimeric enzymes arranged in head-to-tail conformation. Catalysis is performed by two cysteine residues, peroxidatic $\left(S_{p}\right)$ and resolving $\left(S_{r}\right)$. The peroxidatic cysteine reacts with hydrogen peroxide producing a molecule of water and becoming oxidized as sulfenic acid. In a second step the resolving cysteine condenses with the sulfenic acid intermediate so that a molecule of water is produced and both cysteines are linked by a disulfide bridge. For a new catalytic cycle the disulfide has to be reduced. In plant chloroplasts both in vitro and in vivo analyses suggest that NTRC is more relevant for 2-Cys Prx reduction than other plastidial Trxs such as CDSP32 and $\operatorname{Tr} x$

enzymes from other eukaryotic organisms and is predominantly reduced by NTRC. The required reducing power in form of NADPH is produced either by the photosynthetic electron transport chain, which occurs during the day, or from sugars by the initial reactions of the oxidative pentose phosphate pathway, which would be the predominant pathway during the night (Spínola et al., 2008; Cejudo etal., 2012). Though not experimentally established, it is expected that $\operatorname{Trx} x$ and CDSP32 use the reducing power of reduced ferredoxin $(\mathrm{Fd})$ in a reaction catalyzed by Fd-dependent Trx reductase (FTR). Results from our group show that NTRC is unable to reduce plastidial Trxs, such as $\operatorname{Trx} x$ or CDSP32 (Pérez-Ruiz et al., 2006; BernalBayard et al., 2012). Therefore, it seems that the two different pathways, NTRC and FTR/Trx, for 2-Cys Prx reduction are not connected.

\section{CHLOROPLAST 2-Cys Prxs ARE SENSITIVE TO OVEROXIDATION}

The study of the reaction mechanism of chloroplast 2-Cys Prxs revealed that the enzyme may become irreversibly oxidized during the catalytic cycle and shows tendency to form oligomers (Konig et al., 2002). These properties of chloroplast 2-Cys Prxs gained interest when Wood et al. (2003b) proposed the floodgate hypothesis according to which the signaling function of hydrogen peroxide in eukaryotic organisms is due to the overoxidation of the peroxidatic cysteine at the active site of 2-Cys Prxs. As mentioned above, during catalysis the peroxidatic cysteine becomes transiently oxidized to sulfenic acid, which under oxidizing conditions may be overoxidized to sulfinic or even sulfonic acid (Figure 2). This overoxidation inhibits the peroxidase activity of the enzyme thus allowing the local accumulation of hydrogen peroxide, which exerts its function as second messenger (Wood et al., 2003b). Though initially it was thought that overoxidation was an irreversible process, it was then found that sulfiredoxin (Srx) is able to reverse the overoxidized form to the reduced form of the enzyme in a reaction that required ATP and $\mathrm{Mg}^{2+}$ (Biteau et al., 2003; Woo et al., 2003). Overoxidation favors the formation of the HMW form of 2-Cys Prxs, which promotes the chaperone activity of these enzymes (Figure 2). All these data, obtained from analyses with yeast and human enzymes, indicated that the redox status of 2-Cys Prxs is essential to determine their peroxidase or chaperone activity, making them efficient sensors and key components of the response to oxidant conditions (Karplus and Poole, 2012).

In plants, the chloroplast is an essential organelle not only because of photosynthesis, but also because it is the site of synthesis of a variety of compounds, such as hormones, which play a role in signaling. The role of the chloroplast as an important source of hydrogen peroxide is well known (Mubarakshina et al., 2010). Indeed, we have recently shown that restitution of the redox homeostasis exclusively in chloroplasts, by expressing NTRC in the ntrc background mutant under the $R b c S$ promoter, was necessary and sufficient to recover wild type growth and development of lateral roots regardless of the impaired redox homeostasis in root amyloplasts (Ferrández et al., 2012; Kirchsteiger et al., 2012). Therefore, whether or not chloroplast 2-Cys Prxs undergo overoxidation and the mechanisms controlling the redox status of the enzyme are relevant questions to determine their antioxidant and/or signaling function.

Two-dimensional gel electrophoresis analysis of 2-Cys Prx from wild type and mutants deficient in either 2-Cys Prx A or 2Cys Prx B from Arabidopsis revealed the overoxidation of both enzymes (Kirchsteiger et al., 2009). Surprisingly, the NTRC knock out mutant showed lower level of 2-Cys Prx overoxidation than wild type plants, despite the fact that the deficiency of NTRC may cause oxidative stress. This was a first indication suggesting that the reduction of the enzyme, as a pre-requisite for the formation of the sulfenic acid intermediate, is required for the subsequent overoxidation to sulfinic acid, as outlined in Figure 2. The other component affecting the level of 2-Cys Prx overoxidation in chloroplasts is Srx, which is encoded in plants by a single gene, the protein showing dual targeting to chloroplast and mitochondria (Liu et al., 2006; Iglesias-Baena et al., 2011). Chloroplast Srx was shown to effectively reverse 2-Cys Prx overoxidation (Rey et al., 


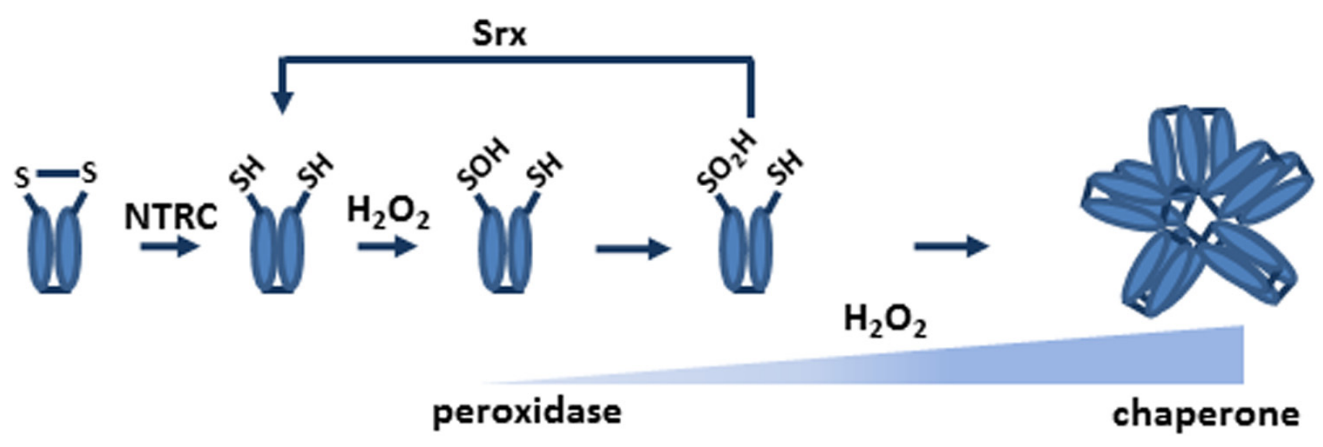

FIGURE 2 | NTRC and Srx determine the redox status of chloroplast 2-Cys Prxs. Under oxidant conditions, the sulfenic acid intermediate of the peroxidatic cysteine residue may be further oxidized to sulfinic acid. The reduction of the enzyme, which is most efficiently performed by NTRC, is a pre-requisite for sulfenic acid formation and, thus, for overoxidation. Srx is able to catalyze the reversion of the overoxidized to the reduced form of the enzyme. Therefore, the redox status of chloroplast 2-Cys Prxs is highly dependent of NTRC and Srx. The quaternary structure of 2-Cys Prxs determines the activity of these enzymes. In the reduced form the enzyme is a dimer and shows peroxidase activity; overoxidation favors the formation of the decameric form, which lacks peroxidase activity and shows chaperone activity.
2007; Iglesias-Baena et al., 2010), though enzyme was also shown to have redox-independent nuclease activity (Chi et al., 2012). The analysis of an Arabidopsis Srx knock out mutant revealed a function of the enzyme in the response to photooxidative stress (Rey et al., 2007). In addition, it was shown that the overoxidation of the chloroplast 2-Cys Prxs, like those of other eukaryotic organisms, triggers the oligomerization of the enzyme, which diminishes the peroxidatic activity while it increases the chaperone activity (Barranco-Medina et al., 2009).

Factors affecting 2-Cys Prx overoxidation in chloroplasts are summarized in the scheme outlined in Figure 2. The reduction of the enzyme, which is predominantly performed by NTRC, is a pre-requisite for the formation of the sulfenic acid intermediate. At high peroxide concentrations this intermediate may become increasingly overoxidized, which switches the peroxidase to chaperone activity of the enzyme. Reversion of the overoxidized form of 2-Cys Prx is catalyzed by Srx in a reaction that requires ATP and $\mathrm{Mg}^{2+}$. According to this scheme, two enzymes, NTRC and Srx, seem to play a central role in controlling the redox status of 2-Cys Prxs in chloroplasts. It has been proposed that 2-Cys Prx may exert a critical function by balancing antioxidant and signaling activities of chloroplast produced hydrogen peroxide (Dietz et al., 2006). This function is probably essential as suggested by the fact that the double knock out mutant lacking both 2-Cys Prx A and B seems not viable. Nevertheless, much effort is still required to determine the reason why these enzymes have such an essential function for plant survival.

\section{THE CYANOBACTERIAL ORIGIN OF CHLOROPLAST 2-Cys Prx OVEROXIDATION}

According to the floodgate hypothesis, the signaling activity of hydrogen peroxide in eukaryotic organisms is based on the inactivation of 2-Cys Prxs by overoxidation, which allows the transient increase in the peroxide necessary to act as second messenger (Wood et al., 2003b). Structural analysis identified the GG(L/V/I)G and YF motifs in sensitive enzymes, and established that the peroxidatic cysteine is $14 \AA$ away from the resolving cysteine, which makes the eukaryotic enzymes about 100-fold more sensitive to overoxidation than the prokaryotic ones (Wood et al., 2003b). Chloroplast 2-Cys Prxs are sensitive to overoxidation (Broin and Rey, 2003; Kirchsteiger et al., 2009; Iglesias-Baena et al., 2010), thus behaving as expected for enzymes of a eukaryotic organelle. Because it is well established that chloroplasts evolved from a prokaryotic endosymbiont (Gould et al., 2008), it arises the question whether 2-Cys Prx sensitivity was already present in the prokaryotic endosymbiont or was a gain-of-function of these enzymes that occurred during chloroplast evolution. To address this question, Pascual et al. (2010) analyzed the presence of the GG(L/V/I)G and YF motifs in the genes encoding 2-Cys Prxs from different sources. This search confirmed the presence of sensitive 2-Cys Prxs, characterized by the presence of both motifs, in eukaryotes. However, it revealed an unexpectedly large number of 2-Cys Prx from prokaryotic organisms containing the GG(L/V/I)G and YF motifs, thus being putatively sensitive to overoxidation. Interestingly, the 2-Cys Prxs from several cyanobacteria, such as Anabaena sp. PCC7120 and Synechocystis sp. PCC6803, were found to contain these motifs. Biochemical analyses revealed that 2-Cys Prx from Anabaena sp. PCC7120 shows a level of sensitivity to overoxidation similar to that of the chloroplast enzymes, whereas 2-Cys Prx from Synechocystis sp. PCC6803 is less sensitive (Pascual et al., 2010). Moreover, in vivo analyses showed different strategies of these cyanobacterial strains to respond to oxidative stress. While Anabaena showed high sensitivity, Synechocystis survived higher concentrations of hydrogen peroxide. The strategy based on high efficiency of hydrogen peroxide detoxification provides higher resistance though, as it is rapidly reduced, the peroxide cannot be used for signaling. In contrast, the Anabaena strategy, based on low capacity of detoxification, causes the increase of hydrogen peroxide required to act as second messenger, though it may have as well a harmful effect. Interestingly, the strategy of chloroplasts, which are equipped with sensitive 2-Cys Prxs and lack catalase, is very similar to the Anabaena strategy. This is in agreement with the proposal that chloroplasts originated from cyanobacterial strains similar to present day Anabaena species (Deusch et al., 2008). 


\section{CONCLUDING REMARKS AND FUTURE PROSPECTS}

The inactivation of the peroxidase activity of 2-Cys Prxs, caused by the overoxidation of their peroxidatic cysteines, has been proposed to be essential for the signaling function of hydrogen peroxide in eukaryotic organisms. In chloroplasts, which constitute an important source of hydrogen peroxide and have a prominent signaling function in plants, 2-Cys Prxs are among the most abundant proteins. Despite the prokaryotic origin of the plant chloroplast, the 2-Cys Prxs of this organelle undergo peroxide-mediated overoxidation, thus behaving as eukaryotic-type enzymes. The redox status of chloroplast 2-Cys Prxs, mostly controlled by NTRC and Srx, may balance the antioxidant and signaling functions of

\section{REFERENCES}

Alkhalfioui, F., Renard, M., and Montrichard, F. (2007). Unique properties of NADP thioredoxin reductase $\mathrm{C}$ in legumes. J. Exp. Bot. 58, 969-978. doi: $10.1093 / \mathrm{jxb} / \mathrm{erl} 248$

Baier, M., Noctor, G., Foyer, C. H., and Dietz, K. J. (2000). Antisense suppression of 2-cysteine peroxiredoxin in Arabidopsis specifically enhances the activities and expression of enzymes associated with ascorbate metabolism but not glutathione metabolism. Plant Physiol. 124, 823 832. doi: 10.1104/pp.124.2.823

Barranco-Medina, S., Lázaro, J. J., and Dietz, K. J. (2009). The oligomeric conformation of peroxiredoxins links redox state to function. FEBS Lett. 583, 1809-1816. doi: 10.1016/j.febslet.2009.05.029

Bernal-Bayard, P., Hervás, M., Cejudo, F. J., and Navarro, J. A. (2012). Electron transfer pathways and dynamics of chloroplast NADPH-dependent thioredoxin reductase C (NTRC). J. Biol. Chem. 287, 33865-33872. doi: 10.1074/jbc.M112.388991

Biteau, B., Labarre, J., and Toledano, M. B. (2003). ATP-dependent reduction of cysteine-sulphinic acid by $S$. cerevisiae sulphiredoxin. Nature 425, 980-984. doi: 10.1038/nature02075

Bréhélin, C., Meyer, E. H., de Souris, J. P., Bonnard, G., and Meyer, Y. (2003). Resemblance and dissemblance of Arabidopsis type II peroxiredoxins: similar sequences for divergent gene expression, protein localization, and activity. Plant Physiol. 132, 20452057. doi: 10.1104/pp.103.022533

Broin, M., Cuiné, S., Aymery, F., and Rey, P. (2002). The plastidic 2cysteine peroxiredoxin is a target for a thioredoxin involved in the protection of the photosynthetic apparatus against oxidative damage. Plant Cell 14, 1417-1432. doi: 10.1105/tpc. 001644

Broin, M., and Rey, P. (2003). Potato plants lacking the CDSP32 plastidic thioredoxin exhibit overoxidation of the BAS1 2-cysteine peroxiredoxin and increased lipid peroxidation in thylakoids under photooxidative stress. Plant Physiol. 132, 1335-1343. doi: $10.1104 /$ pp.103.021626

Cejudo, F. J., Ferrández, J., Cano, B., Puerto-Galán, L., and Guinea, M. (2012). The function of the NADPH thioredoxin reductase C-2Cys peroxiredoxin system in plastid redox regulation and signalling. FEBS Lett. 586, 2974-2980. doi: 10.1016/j.febslet.2012.07.003

Chi, Y. H., Kim, S. Y., Jung, I. J., Shin, M. R., Jung, Y. J., Park, J. H., et al. (2012). Dual functions of Arabidopsis sulfiredoxin: acting as a redoxdependent sulfinic acid reductase and as a redox-independent nuclease enzyme. FEBS Lett. 586, 3493-3499. doi: 10.1016/j.febslet.2012.08.002

Collin, V., Issakidis-Bourguet, E., Marchand, C., Hirasawa, M., Lancelin, J.-M., Knaff, D. B., et al. (2003). The Arabidopsis plastidial thioredoxins. New functions and new insights into specificity. J. Biol. Chem. 278, $23747-$ 23752. doi: 10.1074/jbc.M302077200

Deusch, O., Landan, G., Roettger, M., Gruenheit, N., Kowallik, K. V., Allen, J. F., et al. (2008). Genes of cyanobacterial origin in plant nuclear genomes point to a heterocyst-forming plastid ancestor. Mol. Biol. Evol. 25, 748-761. doi: 10.1093/molbev/msn022

Dietz, K.-J. (2003). Plant peroxiredoxins. Annu. Rev. Plant Biol. 54, 93-107. doi: 10.1146/annurev. arplant.54.031902.134934

Dietz, K.-J. (2011). Peroxiredoxins in plants and cyanobacteria. Antioxid. Redox. Signal. 15, 1129-1159. doi: 10.1089/ars.2010.3657

Dietz, K. J., Jacob, S., Oelze, M. L., Laxa, M., Tognetti, V., de Miranda, S. M., et al. (2006). The function of peroxiredoxins in plant organelle redox metabolism. J. Exp. Bot. 57, 1697-1709. doi: 10.1093/jxb/ erj160

Edgar, R. S., Green, E. W., Zhao, Y., van Ooijen, G., Olmedo, M., Qin, X., et al. (2012). Peroxiredoxins are conserved markers of circadian

chloroplast-produced hydrogen peroxide and, thus, its activity as second messenger. Although much progress has been made on the biochemical properties of 2-Cys Prxs, little is yet known about the mechanisms explaining their function in signaling. The identification of the targets of these enzymes may be of aid to establish these functions.

\section{ACKNOWLEDGMENTS}

Research in our lab was supported by ERDF-cofinanced grants from Ministry of Science and Innovation (BIO2010-15430) and Junta de Andalucía (BIO-182 and CVI-5919).

rhythms. Nature 485, 459-464. doi: 10.1038 /nature 11088

Ferrández, J., González, M., and Cejudo, F. J. (2012). Chloroplast redox homeostasis is essential for lateral root formation in Arabidopsis. Plant Signal. Behav. 7, 1177-1179. doi: $10.4161 /$ psb. 21001

Gould, S. B., Waller, R. F., and McFadden, G. I. (2008). Plastid evolution. Annu. Rev. Plant Biol. 59, 491-517. doi: 10.1146/annurev. arplant.59.032607.092915

Hall, A., Karplus, P. A., and Poole, L. B. (2009). Typical 2-Cys peroxiredoxins - structures, mechanisms and functions. FEBS J. 276, 24692477. doi: 10.1111/j.1742-4658.2009. 06985.x

Iglesias-Baena, I., Barranco-Medina, S. Lázaro-Payo, A., López-Jaramillo, F. J., Sevilla, F., and Lázaro, J. J. (2010). Characterization of plant sulfiredoxin and role of sulphinic form of 2-Cys peroxiredoxin. J. Exp. Bot. 61, 1509-1521. doi: 10.1093/jxb/erq016

Iglesias-Baena, I., Barranco-Medina, S., Sevilla, F., and Lázaro, J. J. (2011). The dual-targeted plant sulfiredoxin retroreduces the sulfinic form of atypical mitochondrial peroxiredoxin. Plant Physiol. 155, 944 955. doi: 10.1104/pp.110.166504

Jang, H. H., Lee, K. O., Chi, Y. H., Jung, B. G., Park, S. K., Park, J. H., et al. (2004). Two enzymes in one: two yeast peroxiredoxins display oxidative stress-dependent switching from a peroxidase to a molecular chaperone function. Cell 117, 625-635. doi: 10.1016/j.cell.2004.05.002

Karplus, P. A., and Poole, L. B. (2012). Peroxiredoxins as molecular triage agents, sacrificing themselves to enhance cell survival during a peroxide attack. Mol. Cell 45, 275-278. doi: 10.1016/j.molcel.2012.01.012

Kirchsteiger, K., Ferrández, J., Pascual, M. B., González, M., and Cejudo, F. J. (2012). NADPH thioredoxin reductase $\mathrm{C}$ is localized in plastids of photosynthetic and non-photosynthetic tissues and is involved in lateral roo formation in Arabidopsis thaliana. Plant Cell 24, 1534-1548. doi: 10.1105/tpc.111.092304

Kirchsteiger, K., Pulido, P., González, M. C., and Cejudo, F. J. (2009). NADPH Thioredoxin reductase $\mathrm{C}$ controls the redox status of chloroplast 2Cys peroxiredoxins in Arabidopsis thaliana. Mol. Plant 2, 298-307. doi: $10.1093 / \mathrm{mp} / \mathrm{ssn} 082$

Konig, J., Baier, M., Horling, F., Kahmann, U., Harris, G., Schurmann, P., et al. (2002). The plant-specific function of 2-Cys peroxiredoxinmediated detoxification of peroxides in the redox-hierarchy of photosynthetic electron flux. Proc. Natl. Acad. Sci. U.S.A 99, 5738-5743. doi: 10.1073/pnas.072644999

Laloi, C., Stachowiak, M., PersKamczyc, E., Warzych, E., Murgia, I., and Appel, K. (2007). Cross-talk between singlet oxygen- and hydrogen peroxide-dependent signaling of stress responses in Arabidopsis thaliana. Proc. Natl. Acad. Sci. U.S.A 104, 672-677. doi: 10.1073/pnas. 0609063103

Lamkemeyer, P., Laxa, M., Collin, V., Li, W., Finkemeier, I., Schöttler, M. A., et al. (2006). Peroxiredoxin Q of Arabidopsis thaliana is attached to the thylakoids and functions in context of photosynthesis. Plant J. 45, 968-981. doi: 10.1111/j.1365313X.2006.02665.X

Liu, X. P., Liu, X. Y., Zhang, J., Xia, Z. L., Liu, X., Qin, H. J., et al. (2006). Molecular and functional characterization of sulfiredoxin homologs from higher plants. Cell Res. 16, 287-296. doi: 10.1038/sj.cr.7310036

Miller, G., Suzuki, N., Ciftci-Yilmaz, S., and Mittler, R. (2010). Reactive oxygen species homeostasis and signalling during drought and salinity stresses. Plant Cell Environ. 33, 453-467. doi: 10.1111/j.13653040.2009.02041.x

Moon, J. C., Jang, H. H., Chae, H. B., Lee, J. R., Lee, S. Y., Jung, Y. J., et al. (2006). The C-type Arabidopsis thioredoxin reductase ANTR-C acts 
as an electron donor to 2-Cys peroxiredoxins in chloroplasts. Biochem. Biophys. Res. Commun. 348, 478-484. doi: 10.1016/j.bbrc.2006.07.088

Mubarakshina, M. M., Ivanov, B. N., Naydov, I. A., Hillier, W., Badger, M. R., Krieger-Liszkay, A. (2010). Production and diffusion of chloroplastic $\mathrm{H}_{2} \mathrm{O}_{2}$ and its implication to signalling. J. Exp. Bot. 61, 3577-3587. doi: $10.1093 /$ jxb/erq171

Muthuramalingam, M., Seidel, T., Laxa, M., Nunes de Miranda, S. M., Gärtner, F., Ströher, E., et al. (2009). Multiple redox and non-redox interactions define 2-Cys peroxiredoxin as a regulatory hub in the chloroplast. Mol. Plant 2, 1273-1288. doi: $10.1093 / \mathrm{mp} / \mathrm{ssp} 089$

Pascual, M. B., Mata-Cabana, A., Florencio, F. J., Lindahl, M., and Cejudo, F. J. (2010). Overoxidation of 2-Cys peroxiredoxin in prokaryotes: cyanobacterial 2-Cys peroxiredoxins sensitive to oxidative stress. $J$. Biol. Chem. 285, 34485-34492. doi: 10.1074/jbc.M110.160465

Pérez-Ruiz, J. M., Spínola, M. C., Kirchsteiger, K., Moreno, J., Sahrawy, M., and Cejudo, F. J. (2006). Rice NTRC is a high-efficiency redox system for chloroplast protection against oxidative damage. Plant Cell 18, 2356-2368. doi: 10.1105/tpc. 106.041541

Petersson, U. A., Kieselbach, T., GarcíaCerdán, J. G., and Schröder, W. P. (2006). The Prx Q protein of Arabidopsis thaliana is a member of the luminal chloroplast proteome. FEBS Lett. 580, 6055-6061.

Pulido, P., Cazalis, R., and Cejudo, F. J. (2009). An antioxidant redox system in the nucleus of wheat seed cells suffering oxidative stress. Plant J. 57, 132-145. doi: 10.1111/j.1365313X.2008.03675.
Pulido, P., Spínola, M. C., Kirchsteiger, K., Guinea, M., Pascual, M. B. Sahrawy, M., et al. (2010). Functional analysis of the pathways for 2-Cys peroxiredoxin reduction in Arabidopsis thaliana chloroplasts. J. Exp. Bot. 61, 4043-4054. doi: 10.1093/jxb/ erq218

Rey, P., Becuwe, N., Barrault, M. B., Rumeau, D., Havaux, M., Biteau, B. et al. (2007). The Arabidopsis thaliana sulfiredoxin is a plastidic cysteinesulphinic acid reductase involved in the photooxidative stress response. Plant J. 49, 505-514. doi: 10.1111/j. 1365-313X.2006.02969.x

Rhee, S. G., Chae, H. Z., and Kim, K. (2005). Peroxiredoxins: a historical overview and speculative preview of novel mechanisms and emerging concepts in cell signaling. Free Radic. Biol. Med. 38, 1543-1552. doi: 10.1016/j.freeradbiomed.2005. 02.026

Rhee, S. G., Woo, H. A., Kil, I. S., and Bae, S. H. (2012). Peroxiredoxin functions as a peroxidase and a regulator and sensor of local peroxides. J. Biol. Chem. 287, 4403-4410. doi: 10.1074/jbc.R111.283432

Serrato, A. J., Pérez-Ruiz, J. M., and Cejudo, F. J. (2002). Cloning of thioredoxin $\mathrm{h}$ reductase and characterization of the thioredoxin reductase-thioredoxin $\mathrm{h}$ system from wheat. Biochem. J. 217, 392-399. doi: 10.1042/BJ20020103

Serrato, A., Pérez-Ruiz, J. M., Spínola, M. C., and Cejudo, F. J. (2004). A novel NADPH thioredoxin reductase, localized in the chloroplast, which deficiency causes hypersensitivity to abiotic stress in Arabidopsis thaliana. J. Biol. Chem. 279, 43821-43827. doi: 10.1074/jbc.M404696200

Spínola, M. C., Pérez-Ruiz, J. M., Pulido, P., Kirchsteiger, K., Guinea,
M., González, M. C., et al. (2008). NTRC: new ways of using NADPH in the chloroplast. Physiol. Plant. 133, 516-524. doi: 10.1111/j.13993054.2008.01088.x

Stacy, R. A. P., Munthe, E., Steinum, T., Sharma, B., and Aalen, R. (1996). A peroxiredoxin antioxidant is encoded by a dormancy-related gene, Per1, expressed during late development in the aleurone and embryo of barley grains. Plant Mol. Biol. 31, 12051216. doi: 10.1007/BF00040837

Stacy, R. A. P., Nordeng, T. W. Culiáñez-Maciá F. A., and Aalen, R. (1999). The dormancy-related peroxiredoxin anti-oxidant, PER1, is localized to the nucleus of barley embryo and aleurone cells. Plant J. 19 1-8. doi: 10.1046/j.1365-313X.1999. 00488.x

Vandenabeele, S., Van Der Kelen, K., Dat, J., Gadjev, I., Boonefaes, T., Morsa, S., et al. (2003) A comprehensive analysis of hydrogen peroxide-induced gene expression in tobacco. Proc. Natl. Acad. Sci. U.S.A 100, 16113-16118. doi: $10.1073 /$ pnas. 2136610100

Vanderauwera, S., Zimmermann, P., Rombauts, S., Vandenabeele, S., Langebartels, C., Gruissem, W., et al. (2005). Genome-wide analysis of hydrogen peroxide-regulated gene expression in Arabidopsis reveals a high light-induced transcriptional cluster involved in anthocyanin biosynthesis. Plant Physiol. 139, 806-821. doi: 10.1104/pp.105. 065896

Woo, H. A., Chae, H. Z., Hwang, S. C., Yang, K. -S., Kang, S. W., Kim, K., et al. (2003). Reversing the inactivation of peroxiredoxins caused by cysteine sulfinic acid formation. Science 300, 653-656. doi: 10.1126/science. 1080273
Wood, Z. A., Schröder, E., Harris J. R., and Poole, L. B. (2003a). Structure, mechanism and regulation of peroxiredoxins. Trends Biochem. Sci. 28, 32-40. doi: 10.1016/S09680004(02)00003-8

Wood, Z. A., Poole, L. B., and Karplus, P. A. (2003b). Peroxiredoxin evolution and the regulation of hydrogen peroxide signalling. Science 300, 650 653. doi: 10.1126/science.1080405

Conflict of Interest Statement: The authors declare that the research was conducted in the absence of any commercial or financial relationships that could be construed as a potential conflict of interest.

Received: 08 July 2013; paper pending published: 17 July 2013; accepted: 24 July 2013; published online: 19 August 2013.

Citation: Puerto-Galán L, Pérez-RuizJM, Ferrández J, Cano B, Naranjo B, Nájera VA, González M, Lindahl AM and Cejudo FJ (2013) Overoxidation of chloroplast 2-Cys peroxiredoxins: balancing toxic and signaling activities of hydrogen peroxide. Front. Plant Sci. 4:310. doi: 10.3389/fpls.2013.00310

This article was submitted to Plant Physiology, a section of the journal Frontiers in Plant Science.

Copyright (C) 2013 Puerto-Galán, PérezRuiz, Ferrández, Cano, Naranjo, Nájera, González, Lindahl and Cejudo. This is an open-access article distributed under the terms of the Creative Commons Attribution License (CC BY). The use, distribution or reproduction in other forums is permitted, provided the original author(s) or licensor are credited and that the original publication in this journal is cited, in accordance with accepted academic practice. No use, distribution or reproduction is permitted which does not comply with these terms. 